\title{
Necessidades em Saúde dos adolescentes na perspectiva dos profissionais da Atenção Primária à Saúde
}

\author{
Health needs of adolescents from the perspective of Primary Health \\ Care professionals
}

Raquel Porto Barros (https://orcid.org/0000-0003-1167-2665) ${ }^{1}$

Paula Regina Costa Mendes de Holanda (https://orcid.org/0000-0002-6102-7413) ${ }^{1}$

Aline Dyelle da Silva Sousa (https://orcid.org/0000-0002-8850-1939) ${ }^{1}$

Maíra Rosa Apostolico (https://orcid.org/0000-0003-2578-8685) ${ }^{2}$

${ }^{1}$ UNINASSAU. R. Guilherme Pinto 114, Graças. 52010-210 Recife PE Brasil.raquel.porto@ sereducacional.com

${ }^{2}$ Autor independente. Atualmente sem vínculo institucional. São Paulo SP Brasil.

\begin{abstract}
The recognition of health needs is essential to develop public actions and policies. $\mathrm{Ob}$ jective: to analyze the recognition and the meeting of the health needs of adolescents, from the perspective of Primary Health Care professionals. Method: qualitative, exploratory and descriptive study, which analyzed 15 interviews with Primary Health Care professionals of the III Health District in the municipality of Recife, state of Pernambuco, Brazil, who have worked in Primary Health Care for a year or more. Content analysis was performed using the webQDA software. Results: five empirical categories emerged: Adolescent and adolescence, Individual and social group needs, Political and normative organization, Professional, team and intersectoral actions and Potentials and limits in adolescent care. The concept of adolescence is based on stereotypes and a fragile articulation to social, economic, political and historical contexts. The professionals recognize the demands compatible with the Primary Care programmatic actions as needs, but they do not have enough instruments to deal with the group peculiarities. The absence of goals in the Municipal Health Plan equals adolescents to other social groups and offers them the same menu of needs. Key words Adolescent, Primary Health Care, Health services needs and demands
\end{abstract}

Resumo $O$ reconhecimento das necessidades em saúde é essencial na elaboração de ações e políticas públicas. Objetivo: analisar o reconhecimento e a satisfação das necessidades em saúde dos adolescentes, a partir da perspectiva de profissionais da Atenção Primária à Saúde. Método: estudo qualitativo, exploratório e descritivo, analisou 15 entrevistas realizadas com profissionais da Atenção Primária à Saúde que atuam no Distrito Sanitário III em Recife, Pernambuco, Brasil, há um ano ou mais. Foi realizada análise de conteúdo com apoio do software webQDA. Resultados: emergiram cinco categorias empíricas: Adolescente e adolescência, Necessidades individuais e do grupo social, Organização política e normativa, Ações profissionais, em equipe e intersetoriais e Potencialidades e limites no atendimento ao adolescente. A compreensão sobre a adolescência é pautada em estereótipos e de frágil articulação aos contextos sociais, econômicos, políticos e históricos. Os profissionais reconhecem como necessidades as demandas compatíveis com as ações programáticas da Atenção Básica sem terem instrumentos suficientes para lidar com as peculiaridades do grupo. A ausência de metas no Plano Municipal de Saúde iguala os adolescentes a outros grupos sociais e lhes oferece $o$ mesmo cardápio de necessidades.

Palavras-chave Adolescente, Atenção Primária à Saúde, Necessidades e demandas de serviços de saúde 


\section{Introdução}

A atenção à adolescência requer planejamento e organização com a colaboração de diferentes atores sociais. As mudanças demográficas do Brasil têm desacelerado o crescimento da população adolescente, embora seja ainda a maior de toda a história do país. No Brasil, adolescente é o indivíduo com idade entre 10 e 19 anos, que vive majoritariamente em áreas urbanas, contribui para a aceleração da economia e está vulnerável a violência, gravidez precoce, uso e abuso de drogas, agravos sexualmente transmissíveis. Têm enfrentado ainda, uma distorção entre idade, escolaridade e dificuldades de acesso ao mercado de trabalho'.

A atenção integral deve contemplar o fortalecimento das ações de promoção da saúde e a reorientação dos serviços, ampliando a capacidade de resposta às necessidades. Segundo as Diretrizes Nacionais para Atenção Integral ao Adolescente ${ }^{1}$, é por meio de um modelo de atenção local formulado de acordo com especificidades regionais que se respondem às necessidades em saúde. A integralidade do cuidado deve ser o objetivo da rede de atenção à saúde como um todo, rompendo barreiras financeiras, geográficas, culturais e simbólicas que impedem o jovem de acessar os serviços de saúde².

Em Recife, município cenário deste estudo, o Plano Municipal de Saúde (PMS) de 2018-2021 requisitou um amplo debate social acerca das políticas de saúde locais. Embora o PMS aponte um conjunto de metas, a população adolescente não foi destacada na proposta ${ }^{3}$. A despeito do que foi definido no PMS, Recife apresenta uma taxa de mortalidade adolescente elevada em comparação com outros estados. Complementarmente, a violência interpessoal envolvendo adolescentes somou 23\% das notificações, no período de 2009 a $2018^{3}$.

Diante do contexto apresentado, este estudo tomou como questão de investigação: como os profissionais da Atenção Primária à Saúde percebem o adolescente, reconhecem necessidades e realizam suas ações em saúde, em vista das fragilidades das políticas públicas locais? Tomou-se como objeto o reconhecimento e a satisfação das necessidades em saúde dos adolescentes.

A finalidade do estudo é identificar características para o planejamento de um cuidado integral ancorado nas necessidades em saúde. A carência de informações sobre a população adolescente, em suas dimensões social, cultural, afetiva, política e a ausência de estratégias que priorizem a atenção integral ao adolescente justificam a realização do estudo.

O objetivo do estudo foi analisar o reconhecimento e a satisfação das necessidades em saúde dos adolescentes, a partir da perspectiva de profissionais da Atenção Primária à Saúde.

\section{Método}

Trata-se de um estudo descritivo e exploratório, de abordagem qualitativa, sobre as necessidades em saúde dos adolescentes, parte de um projeto maior sobre as políticas, as instituições e os profissionais para atenção integral à saúde da criança e do adolescente ${ }^{4}$. Para garantir a qualidade do estudo, foram seguidas as diretrizes para pesquisas de abordagem qualitativa previstas no check list Consolidated criteria for reporting qualitative research (COREQ) $)^{5}$.

O estudo foi aprovado pelo Comitê de Ética em Pesquisa e autorizado pela Secretaria Municipal de Saúde de Recife. Todos os aspectos éticos foram observados, os participantes assinaram o Termo de Consentimento Livre e Esclarecido e as entrevistas foram codificadas, garantindo o anonimato.

O referencial teórico e metodológico utilizado foi a Teoria da Intervenção Práxica da Enfermagem em Saúde Coletiva (TIPESC), metodologia dinâmica e participativa, fundamentada na visão de mundo materialista histórica e dialética. Foram desenvolvidas as duas primeiras etapas da TIPESC que consistem na captação e na interpretação da realidade objetiva, nas três dimensões do fenômeno da realidade: estrutural, particular e singular ${ }^{6}$.

Caracterizar as dimensões de uma realidade permite identificar processos protetores e de desgastes e compreender como a saúde e a doença são determinadas, incluindo a expressão de vulnerabilidades e necessidades em saúde. A compreensão sobre as dimensões da realidade objetiva instrumentalizar para a construção de projetos de intervenção resolutivos, com abertura de novos processos modificadores da realidade de saúde do indivíduo e da coletividade ${ }^{6,7}$.

No fenômeno necessidades em saúde dos adolescentes, a dimensão estrutural consiste nas políticas públicas nacionais, estaduais e municipais, que diretamente imprimem sobre os jovens uma determinada maneira de ser e estar na sociedade; a dimensão particular consiste na organização dos serviços em saúde e na articulação da rede de atenção, que em última instância de- 
termina o acesso dos jovens aos serviços; na dimensão singular está a compreensão dos profissionais sobre a adolescência, adolescente e como percebem, acolhem e satisfazem as necessidades em saúde. Neste estudo, a dimensão singular foi priorizada e a categoria necessidades em saúde adotada para análise dos resultados.

As necessidades em saúde não se restringem às demandas biológicas, como problemas de saúde ou doenças, sofrimentos ou riscos. É constituída também pelas carências ou vulnerabilidades relacionadas ao modo de vida e identidade, expressos na condição necessária para o gozo da vida e ideais de saúde ${ }^{8}$, tais como os aspectos sociais e ambientais como moradia, alimentação, educação, emprego e ambiente ${ }^{8}$.

É nas relações de reprodução social que os sujeitos se desenvolvem como seres sociais e as necessidades em saúde se originam. As relações de produção e reprodução são a base das necessidades em saúde dos indivíduos determinando, assim, o processo saúde doença. Para reconhecer essas necessidades deve-se utilizar instrumentos epidemiológicos potentes para captar a produção de diferentes grupos sociais ${ }^{9}$, além de competência ética e política que permita ao profissional reconhecer o que é necessidade em saúde.

O cenário foi o Distrito Sanitário III de Recife, que conta com uma rede de APS composta por oito equipes dispostas em sete Unidades de Saúde da Família (USF), uma equipe do Núcleo de Apoio à Saúde da Família (NASF), seis equipes de Saúde Bucal e seis equipes do Programa de Agentes Comunitários de Saúde (PACS). Todos os profissionais com formação superior (enfermeiro, médico, psicólogo, odontólogo, fisioterapeuta, farmacêutico e nutricionista) das USF e NASF foram convidados a participar, totalizando uma população de 51 profissionais e estabelecendo-se o limite de conveniência de uma equipe por USF para a coleta de dados.

Os critérios de inclusão foram: atuação na APS, há um ano ou mais, com atendimento a adolescentes. A coleta de dados foi feita pelas pesquisadoras, residentes no município, treinadas para a realização das entrevistas e atuantes em uma instituição de Ensino Superior que mantém atividades práticas no Distrito. Elas foram apresentadas aos participantes momentos antes da entrevista, por um profissional da UBS, que auxiliou no recrutamento dos profissionais. Os participantes foram entrevistados pessoalmente, em espaço reservado e na presença apenas das pesquisadoras, por aproximadamente 20 minutos, utilizando gravador digital e registro de notas de campo. $\mathrm{O}$ roteiro de entrevista consistiu em uma caracterização dos participantes e questões norteadoras sobre a atenção ao adolescente na APS.

Foram realizadas 15 entrevistas e outros seis profissionais se recusaram a participar, sob alegação de desinteresse no estudo ou indisponibilidade. Todo o conteúdo foi transcrito por duas pesquisadoras e validado pelos participantes que autorizaram a utilização das entrevistas, sem necessidade de refazer ou modificar o conteúdo.

Para apoio à análise qualitativa, foi utilizado o software webQDA e as técnicas de análise de conteúdo ${ }^{10}$. A codificação foi realizada e revisada pelas pesquisadoras e para a análise, as entrevistas foram classificadas segundo a caracterização dos participantes. Foram estabelecidos códigos em árvore para a análise do conteúdo, definidos a priori a partir das temáticas propostas no roteiro semiestruturado. Outros recursos do software como palavras mais frequentes, nuvem de palavras e questionamentos por matrizes foram aplicados, buscando a relação entre os conteúdos das entrevistas e as características dos participantes. O cruzamento de dados a partir das matrizes evidenciou resultados não perceptíveis na análise manual.

\section{Resultados}

Participaram do estudo 11 mulheres e quatro homens, todos com mais de 30 anos de idade e a maioria casados. Sete entrevistados eram enfermeiros, três médicos e os demais eram odontólogo, psicólogo, fisioterapeuta, nutricionista e assistente social; todos desempenhavam atividades assistenciais. Seis entrevistados tinham especialização como maior nível de formação, oito cursaram mestrado e apenas um tinha somente a graduação.

Um terço dos participantes tinha filhos sendo os de três de idade inferior a 12 anos, dois entre 12 e 18 anos e cinco com 19 anos ou mais, indicando que metade dos participantes já vivenciou a adolescência com os próprios filhos. Quanto ao sexo dos filhos, cinco participantes são pais de meninos, dois são pais de meninas e três são pais de meninos e meninas.

Oito participantes referiram ter feito cursos ou formações na temática da saúde do adolescente e todos atuam na APS há mais de cinco anos. Quanto ao tempo na função atual, três estão há menos de cinco anos, oito entre cinco e nove anos e quatro trabalham na mesma função há pelo menos 10 anos. 
Os resultados foram organizados em cinco categorias empíricas.

\section{Adolescente e adolescência}

Todos os entrevistados caracterizaram o adolescente. O recurso de descritores do webQDA permitiu distinguir entre características positivas ou negativas e identificar que as características positivas foram provenientes apenas das entrevistas com enfermeiros e médicos.

Os adolescentes foram descritos como saudáveis e que procuram a USF diante de alguma queixa específica, iniciantes na atividade sexual, desconhecedores das doenças e necesitados de muito apoio, pois são carentes de informação. Alguns têm interesse em participar de atividades da USF quando se tem o apoio da família, seja em casos de agravos ou prevenção.

Por outro lado, um conjunto de fontes se referiu aos adolescentes como imaturos e fragilizados, desinteressados, inconsequentes, ociosos e com hábitos alimentares ruins. Os entrevistados citam ainda a cobrança social excessiva sobre alguém de pouca idade e a marginalização da adolescência.

\section{Necessidades individuais e do grupo social}

Os entrevistados associaram as demandas trazidas ao serviço às necessidades em saúde, sendo mais recorrentes aquelas relacionadas à sexualidade, prevenção ou diagnóstico de infecções sexualmente transmissíveis, busca pelo planejamento familiar ou pré-natal, questões relacionadas à obesidade, cuidados odontológicos, violência doméstica, problemas e conflitos familiares, busca pela atenção em saúde mental, que rotineiramente são encaminhadas para os psicólogos do NASF ou para os Centros de Apoio Psicossocial (CAPS) do território.

eles [adolescentes] estão muitos expostos, muito vulneráveis, abertos a tudo, suscetiveis a tudo, as drogas estão no meio deles, a violência [...], o álcool [...]. Se eles não estão transando, tomando álcool, bebendo, fumando, eles não se enquadram dentro do padrão. Então isso é muito perigoso, muito delicado, muito sério. Então imagine como é que vai ser o adolescente, futuro adulto e uma pessoa que hoje já tá nesse contexto [...]. (E4)

Outras necessidades citadas não são percebidas como demandas de saúde, incluindo as relacionadas ao contexto social, econômico, familiar e territorial.
O problema vai na questão social [...] já que os sistemas eles não estão articulados e a gente enquanto Saúde da Família, enquanto Atenção Básica, a gente não tem pernas para alcançar. (E7)

\section{Organização política e normativa}

Esta categoria identificou como os profissionais articulam as diretrizes do município com a satisfação das necessidades em saúde reconhecidas. Ressaltaram a importância da organização política e das normativas que respaldam as ações em saúde. Porém, a falta de um planejamento específico para o público adolescente e a falta de incentivo para as equipes de saúde geram consequências e o acesso do adolescente ao serviço de saúde fica comprometido.

[...] existe uma politica de saúde da criança, [...] a gente vê que tem outubro rosa, novembro azul, mas não existe nada direcionado à adolescência. Não existe nenhuma ação, embora a gente sabe (sic) que o dia a dia é muito mais importante do que uma ação. Mas eu acho que é muito falho o sistema, não só o município, acho que a nível nacional mesmo, de investimento nesses adolescentes, de uma forma geral. (E10)

Outros profissionais desconhecem políticas públicas de saúde para o adolescente e se queixam da precariedade da rede de serviços para referência. Citaram a assistência descontinuada que fragiliza um dos princípios fundamentais da APS que é o vínculo. Não há uma programação específica, mas apenas atendimento da demanda espontânea.

$O$ atendimento do adolescente é encaixado dentro das outras políticas, na política de saúde da mulher e na saúde sexual e reprodutiva. (E8)

Alguns entrevistados citaram a necessidade de ampliação da rede de atenção à saúde do adolescente, a falta de estrutura e apoio para as equipes de saúde, a escassez de recursos físicos, a agenda lotada e a falta de tempo como entraves para a implantação eficiente de ações. Tal situação compromete o acesso dos jovens nas redes de saúde de modo satisfatório e eficaz.

$\mathrm{Na}$ prática quando a gente se depara com a situação, a gente fica travado na atenção básica e com o problema na mão, porque não tem pra onde mandar. A rede tem [...] mas não é suficiente para dar conta da demanda. [...] Então a gente precisaria ampliar essa rede de atenção, tanto dentro da Atenção Básica quanto na Atenção Secundária e Terciária. (E7) 


\section{Ações profissionais, em equipe e intersetoriais}

Esta categoria buscou analisar as estratégias utilizadas pelos entrevistados para a satisfação das necessidades dos adolescentes, pautadas no trabalho individual, da equipe ou intersetorial. Foram enfatizados pontos positivos, como acolhimento, imunização, consulta médica, consulta de enfermagem, atendimento odontólogo, acompanhamento com os agentes de saúdes e busca pela articulação entre a equipe.

Os nossos serviços são porta aberta e aí quando o adolescente vem ao acolhimento ou quando o agente de saúde identifica, numa visita domiciliar, a gente procura fazer o máximo possível pra dar esse suporte, principalmente nas questões da sexualidade e nas questões psicológicas. (E7)

Outros ressaltaram pontos negativos como a inexistência de grupos específicos para os adolescentes e um cronograma de atendimento. A falta de condições para satisfazer as necessidades em saúde ou oferecer atendimento resolutivo foram atribuídas à iniciativa e engajamento pessoal do profissional, desarticulado de um contexto de políticas públicas.

Se ela [equipe] se empenhar ela faz qualquer trabalho, basta querer, entendeu? Eu acho que o que está deixando a desejar é isso. As pessoas elas não tão mais com aquela garra, aquele interesse que existia quando eu entrei. A gente fazia eventos, botava tudo no meio do sol, entendeu? [...] não adianta você trabalhar com adolescente se você não se identifica com adolescente. (E3)

A falta de parcerias ou articulações intersetoriais gera preocupação principalmente em relação à referência e contra referência. Alguns expressaram interesse em buscar parcerias dentro do território e com outros setores, identificando objetivos comuns e maior efetividade nas ações previstas.

Eu acho que uma das coisas que eu sinto mais necessidade é a questão da fragilidade da rede, de como as coisas demoram para serem conseguidas. $E$ o envolvimento mesmo do conselho tutelar, do Ministério Público e a gente vê situações que precisam de ser feitas imediatamente. (E11)

O Programa Saúde na Escola (PSE) se destacou por contribuir no desenvolvimento de ações, aumentar o acesso ao público adolescente pela APS e uma oportunidade de ajudar o adolescente por conseguir concretizar atividades na escola e trabalhar temas pertinentes à adolescência.

$O$ vinculo que eu tenho aqui com a escola municipal, que é que a gente presta o programa de PSE e que a gente vai rotineiramente, e a presença deles, dos que estão na escola, realmente eles são bem ordenados até pela diretoria, em questão de falta, por uma rebeldia, eles [adolescentes] estão sempre controlados. (E2)

\section{Potencialidades e limites no atendimento ao adolescente}

As potencialidades estão localizadas na USF, representadas pelo acesso, acolhimento, marcação das consultas, livre demanda e a confiança depositada na equipe, capazes de ampliar o campo de cuidado e aumentar a oferta de serviços nas áreas de abrangência das ESF. Os profissionais destacaram facilidades para consultas na USF com dentista e enfermeiro, atendimento para a prevenção ginecológica e acolhimento, momentos importantes para a escuta, exatamente por possibilitar o atendimento de necessidades em saúde que não estão diretamente ligadas ao corpo biológico. Entretanto, o conteúdo de algumas entrevistas descreve uma satisfação plena de necessidades em saúde em contradição aos problemas relatados quanto à estrutura e aos recursos do serviço e do município.

Veja, eles [adolescentes] são atendidos e toda a necessidade que vem deles são sanadas. Qualquer adolescente que chega aqui consegue marcar consulta para mesma semana [...] não tem demora de consulta, ou com enfermeira, ou com a médica [...] e quando vem a gente faz a abordagem do todo, a gente faz exame, ainda orienta algumas situações específicas e encaminha para o profissional mais específico, quando vê a necessidade. Mas isso é muito da demanda espontânea, da busca deles, se eles não buscarem, de fato não existe o atendimento. (E4)

Como limitação, há uma preocupação com a ineficiência da rede, dos encaminhamentos e a falta de vagas para consultas especializadas em ambulatórios. A falta de uma política pública direcionada ao adolescente e as dificuldades de acolhimento de casos de maior complexidade também impactam no atendimento. Percebe-se uma ruptura do modelo de atenção que passa do final da infância para a idade adulta, sem contemplar os adolescentes como grupo prioritário.

Justamente isso, a falta de uma política específica para você estar [...] apoiando esses adolescentes. Acho que falta muito isso, uma politica mais eficaz e eficiente para atender essa demanda que é muito esquecida, infelizmente. (E10)

A falta de estrutura na USF, a rotina intensa pela alta demanda de trabalho e a falta de profissionais especializados tiveram destaque, den- 
tre outros aspectos que dificultam as ações de promoção da saúde e prevenção de agravos. Da estrutura dos serviços, segue a dificuldade dos profissionais em encontrar ações de educação permanente. As falas exemplificam o despreparo sentido pela equipe de saúde para atender os adolescentes, que culmina no desinteresse pela temática.

Porque infelizmente o problema é nacional, político, social e a gente não consegue enquanto equipe de saúde, enquanto Atenção Básica, a gente não consegue avançar [...]. E dentro da Saúde da Família a gente tem muitas limitações. Por várias questões, pela demanda, pela capacitação profissional, que a gente sabe que é importante [...]. A gente precisa de profissionais especializados, que tenham uma condição de dar um suporte maior, [...] até serviços pra esses adolescentes que eles possam estar utilizando. (E7)

Outros profissionais se queixaram da falta de adesão dos adolescentes e muitos atendimentos são realizados por demanda, com foco na queixa.

A dificuldade [...] é justamente [...] adesão ao tratamento ou às orientações. Você pode dar o encaminhamento, e estar agendado pra tal data, com tal profissional, em tal lugar. E isso deu um trabalho tremendo pra conseguir, e ele [adolescente] não comparece. (E13)

\section{Discussão}

Importante iniciar essa discussão reforçando que a integralidade do cuidado é o alicerce para uma melhor qualidade das ações de promoção da saúde, prevenção, recuperação e reabilitação ${ }^{11}$.

Os entrevistados reconheceram potencialidades e dificuldades que comprometem o atendimento integral dessa população. Há uma relação circular entre a produção de serviços de saúde e a satisfação de necessidades que ocorre por meio do consumo das ações ${ }^{8}$. Neste sentido, a ausência de ações específicas aos adolescentes no Plano Municipal de Saúde transparece na fala dos participantes, que reconhecem as demandas abarcadas pelas ações programáticas da APS como necessidades, ainda que limitadas, sem, contudo, terem instrumentos potentes para lidar com as peculiaridades dessa população.

A característica dos participantes, atuantes há mais de cinco anos na APS, com capacitações para saúde do adolescente e titulação em nível de mestrado mostra uma estrutura de recursos humanos em saúde potencialmente preparada para desenvolver ações efetivas e transformadoras dos territórios. Carecem, entretanto, de direcionamentos normativos, políticas públicas municipais e respaldo técnico.

Alguns entrevistados ressaltaram o desconhecimento dos adolescentes sobre os cuidados com a própria saúde. Destaca-se que a construção do conhecimento para o cuidado em saúde com o adolescente deve pautar-se em estratégias lúdicas, tais como músicas, dramatizações e vídeos, valorizando a vivência educativa em grupo, construção de conhecimento compartilhado, fortalecimento do vínculo com a equipe de saúde e consequentemente maiores possibilidades de intervenção ${ }^{12}$.

Ao analisar os resultados sob o enfoque geracional é pertinente considerar que, assim como as vivências ocorrem de maneiras distintas, as gerações também não são homogêneas. Há influência do ritmo biológico, mas este não define uma geração sozinho ${ }^{13}$. Dessa forma, os profissionais estão diante de usuários que demandam uma atenção específica e a compreensão dos aspectos geracionais não emergiram nas entrevistas. $\mathrm{O}$ embate geracional ocorre entre profissionais e adolescentes, também no reconhecimento de vulnerabilidade e satisfação de necessidades e, consequentemente, na afinidade para propor intervenções. As vivências relacionadas ao processo saúde doença dos adolescentes precisam ser exploradas e valorizadas na formulação das ações, estratégias e políticas públicas, contrapondo-se ao modelo prescritivo e impositivo de atenção à saúde.

$\mathrm{Na}$ fase marcada por vulnerabilidades, os adolescentes se encontram expostos a violência, acidentes, negligência, desproteção, abandono afetivo-social, moradia inadequada, exclusão social, questões relativas à sexualidade, dificuldade no acesso a cultura, educação e a serviços de saú$\mathrm{de}^{14}$.

Os entrevistados citam a importância dos aspectos sociais, mas pouco articulam suas ações a esse contexto. É fundamental considerar os aspectos históricos, sociais e culturais para compreender suas necessidades e como partícipes de sua própria história e agentes de transformação na sociedade. Para tanto, os adolescentes devem ser percebidos como sujeitos sociais, críticos, que possam exercer participação e autonomia para a formação de sua cidadania e a consolidação de valores ${ }^{15}$. O engajamento das equipes na elaboração de estratégias participativas e emancipatórias é um caminho potente a ser trilhado.

Pesquisadores dinamarqueses analisaram registros de queixas inespecíficas de pré-ado- 
lescentes, consideradas um indicador de baixo bem estar. Concluíram que crianças com queixas inespecíficas frequentes e baixa autoavaliação usam mais os serviços de atenção primária do que crianças sem queixas ou com autoavaliação boa. Destacam ainda que o aumento das queixas inespecíficas é um fenômeno global e que requer intervenções precoces para evitar morbidades e aumento de custos em saúde ${ }^{16}$. Frente aos resultados do presente estudo, a estrutura para ações de monitoramento e prevenção é uma necessidade urgente. Os relatos apontam para a atuação prioritariamente na demanda espontânea, com ações curativas e limitadas.

As ações focadas na atenção ao corpo biológico foram mais facilmente percebidas como necessidades em saúde, pelos participantes. Os profissionais apontaram dificuldades para intervir nas situações de saúde complexas, seja pela falta de preparo ou pela ausência de recursos. Estudo que explorou a perspectiva de pais de adolescentes sobre as vulnerabilidades identificou relação com a exposição às drogas, ao sexo precoce, aos hábitos alimentares inadequados e às necessidades de acesso aos serviços de saúde. Embora os pais priorizem as consultas médicas e odontológicas, as ações educativas são valorizadas e consideradas importantes na atenção à saúde dos adolescentes ${ }^{17}$.

$\mathrm{Na}$ perspectiva dos adolescentes estudantes de escolas públicas, o estilo de vida foi o demandante da atenção à saúde, descritos positivamente pelo bem-estar físico e psicológico, alimentação, atividade física e, negativamente, pela vulnerabilidade representada no uso e abuso de drogas. $\mathrm{O}$ adolescente, a despeito dos rótulos e estereótipos reforçados cotidianamente, tem visões de mundo imbricadas em posições críticas e políticas, influenciadas pelo estilo de vida e não cabem nos protocolos institucionais que não consideram a escuta e a participação como elementos prioritários $^{18}$.

Pesquisa realizada no nordeste brasileiro identificou que a população jovem de um território atendido pela Estratégia Saúde da Família (ESF) procura pelos serviços da APS e participa três vezes mais de ações educativas e de prevenção do que a população fora da área de cobertura. As áreas com cobertura da ESF são mais vulneráveis socialmente e, portanto, as ações atendem para além de queixas biológicas e condizem com os princípios da ESF, indicando potencial para a equidade ${ }^{19}$. No presente estudo, o vínculo e o acesso dos adolescentes ao serviço, descritos como pontos positivos, são uma potencialdiade para a estruturação da atenção focalizada nas necessidades em saúde do grupo.

Em outro sentido, estudo realizado no sudeste brasileiro buscou discutir as dificuldades dos adolescentes em perceber a USF como espaço para produção e promoção da saúde. A ausência dos adolescentes no serviço foi explicada pela falta de acolhimento e vínculo. Destaca-se a necessidade do serviço de saúde superar a visão patologizante da adolescência, sempre caracterizada como um período turbulento, de crises e rebeldia, e estabelecer um compromisso com o adolescente, valorizando seu conhecimento ampliado sobre a saúde e sobre o território e sua capacidade de contribuir no planejamento do cuidado ${ }^{2}$.

Os participantes reconheceram que existem necessidades mais amplas do que aquelas expressas como demandas, mas não apontaram instrumentos potentes para legitimar essas necessidades e satisfazê-las, voltando sempre ao enfoque biopsicossocial. A percepção problemática da adolescência é agravada pelos discursos sobre o adolescente como sujeito inapto, despreparado e incompleto, de pronunciamento inválido e de necessidades invisíveis. A adolescência como categoria geracional não pode ser analisada somente pelo enfoque cronológico, embora essa seja uma abordagem frequente na literatura, conforme apontam autores de uma revisão integrativa sobre violência entre parceiros íntimos adolescentes $^{20}$.

Observou-se a importância de vínculo, diálogo e apoio familiar nas questões relacionadas à sexualidade. Estudo realizado no sul do Brasil descreveu a importância da família da gestante adolescente, como ponto de apoio fundamental. Embora as adolescentes tenham apoio inicial de amigos e pessoas não ligadas à família, é nos pais e parentes mais próximos que $\mathrm{o}$ apoio se efetiva ${ }^{21}$.

Um caminho para reverter a reificação das necessidades, tida como o processo de naturalização e descontextualização histórica das demandas, é a busca por formas sociais de superação ${ }^{8}$. A transposição do foco no adolescente pela categoria adolescência torna-se um caminho viável. Ao ampliar as ações individuais para o coletivo, com estratégias participativas, agregadoras, emancipadoras, informativas e construtivas é possível aproximar o serviço de saúde das expectativas e necessidades dos adolescentes.

O processo de trabalho das equipes tem apresentado resultados insatisfatórios, como observado em estudo realizado em Minas Gerais. Os componentes relacionados ao cuidado e ao mo- 
nitoramento das condições de saúde pela APS mostraram-se insuficientes em relação à atuação no território ${ }^{22}$.

Estudo de revisão desenvolvido por pesquisadores chineses, sobre políticas de saúde mental para adolescentes em países de baixa ou média renda, identificou aspectos que definem a entrada da temática nas agendas políticas locais: problemas como a baixa consciência e vontade política, falta de priorização dos problemas da população adolescente frente a outros agravos que afetam adultos, a relutância dos profissionais em participar de debates políticos, a incapacidade legal dos adolescentes de advogar em seu favor e defender direitos e necessidades, valores sociais e culturais traduzidos em estereótipos sobre adolescência, entre outros. Destacam os autores que a participação de organizações não governamentais supre ou minimiza a ausência de serviços de assistência, mas por outro lado, obscurecem a urgência de políticas públicas, favorecendo a fragmentação das ações e planejamentos locais ${ }^{23}$.

A intersetorialidade é um ato complexo, mas importante como uma estratégia política para expandir as ações com qualidade. O PSE foi citado como potencial meio para alcançar os adolescentes. Os valores e as culturas organizacionais de ambos os serviços são diferentes e devem ser considerados no planejamento de ações conjuntas, para alcance de resultados efetivos. Os acordos e as colaborações com novos parceiros são essenciais para a produzir condições de cuidado, proteção e bem-estar para os adolescentes ${ }^{24}$. As ações em saúde promovidas no espaço escolar podem fortalecer o protagonismo dos adolescentes, embora dependam da conjugação de saberes, condições estruturais, organização dos setores e espaços envolvidos, das equipes e dos profissionais ${ }^{25}$.

Os serviços dos entrevistados não têm uma estrutura favorável para o acesso e a permanência dos adolescentes. Dentre os motivos para a não procura do serviço de saúde apontados por adolescentes da Bahia estão barreiras geográficas e organizacionais que envolvem inexistência do atendimento procurado, insatisfação com o atendimento prestado ou acesso a planos privados de saúde que suprime a busca pela APS ${ }^{19}$. A falta de recursos físicos foi relatada. Para efetiva integralidade, o conhecimento sobre a realidade da comunidade adstrita e o trabalho coletivo devem ser ampliados por meio do trabalho em equipe multiprofissional, transdisciplinar e intersetorial, superando o caráter pontual das ações ${ }^{26}$.

Os entrevistados destacaram o acolhimento como uma atividade fragmentada, que não se integra às demais e não favorece o cuidado. $\mathrm{O}$ acolhimento, a ação comunicacional, o ato de receber e escutar a população que busca pelas redes de saúde oportuniza respostas adequadas a cada demanda e em todo o trajeto da busca ${ }^{27}$. $\mathrm{O}$ acolhimento favorece as relações entre os profissionais de saúde e a clientela adolescente, ao procurarem pela APS em qualquer situação, mas pressupõe disposição, organização e preparação da equipe para avaliar e atender, buscando a máxima resolubilidade possível ${ }^{28}$.

$\mathrm{O}$ encaminhamento à rede foi considerado deficitário, com exceção do CAPS, serviço de referência para as demandas de saúde mental. Embora a APS seja a porta de entrada da rede e o matriciamento responsável pelos encaminhamentos e monitoramento, estudo realizado em um município de Minas Gerais identificou que a deficiência da rede tem como consequência direta o isolamento intersetorial e intrasetorial do CAPS, que passa a adotar posição central na Atenção à Saúde Mental, desarticulando-se da APS ${ }^{29}$.

A falta de engajamento dos profissionais foi questionada pelos entrevistados e a iniciativa pessoal atribuída como forma de alcançar resultados positivos na assistência. Entretanto, a perspectiva da integralidade na atenção a populações especiais depende não somente dos profissionais que realizam ações assistenciais, mas também dos gestores, responsáveis pelo planejamento de recursos humanos e materiais dos serviços ${ }^{30}$.

\section{Considerações finais}

O presente estudo buscou analisar o reconhecimento e a satisfação das necessidades em saúde dos adolescentes, na perspectiva de profissionais da APS. Os resultados evidenciaram que a compreensão sobre a adolescência é pautada em estereótipos e de frágil articulação aos contextos sociais, econômicos, políticos e históricos, limitando o reconhecimento e a satisfação das necessidades em saúde. Os espaços de produção de saúde devem incorporar ao planejamento a formação, realizar debates acerca da adolescência como categoria geracional, compreender a determinação de saúde e doença, os potenciais de fortalecimento e desgastes que possam ancorar intervenções transformadoras.

Por si só, a adolescência é um período de transformações significativas individuais, mas que depende de estratégias coletivas para a satisfação das necessidades. Os adolescentes constituem uma categoria e isso os torna potentes para 
o crescimento coletivo e vulneráveis aos impactos da conjuntura macrossocial. A vulnerabilidade, por sua vez, é sentida e reconhecida pelos serviços de saúde como uma demanda individual, dificultando a modificação dos potenciais de desgaste do coletivo.

As necessidades relatadas pelos participantes revelam a limitação que o Plano Municipal de Saúde imprime no processo de trabalho em saúde. A ausência de metas específicas para os adolescentes os colocam em igualdade aos demais usuários dos serviços, oferecendo a eles o mesmo cardápio de necessidades. Questiona-se se são os adolescentes invisíveis ou se os serviços de saúde, desde sua política instauradora, incapazes de reconhecer essa população, suas necessidades e satisfazê-las.

\section{Colaboradores}

MR Apostolico trabalhou na concepção da pesquisa, na metodologia, na análise, nas conclusões e na redação final; RP Barros, PRCM Holanda e ADS Sousa atuaram na pesquisa, na análise, na metodologia, nas conclusões e na redação final.
Assim, as demandas relatadas relacionadas ao planejamento familiar, às ISTs e aos conflitos familiares, embora reconhecidas, não extrapolam o cotidiano das ações em saúde, enquanto outras temáticas pertinentes aos adolescentes escapam pela impossibilidade de satisfação.

O estudo apresentou como limitação a participação de um único distrito do município. As entrevistas, embora esclarecedoras, careceram de aprofundamento de alguns tópicos, como outras necessidades discutidas na literatura e que foram pouco citadas, como violência, uso e abuso de álcool e outras drogas, marginalização e envolvimento no crime, justificando estudos de continuidade, para desvelar novos conhecimentos e uso dos recursos disponíveis no software adotado.

\section{Referências}

1. Brasil. Ministério da Saúde (MS). Diretrizes nacionais para a atenção integral à saúde de adolescentes e jovens na promoção, proteção e recuperação da saúde. Brasília: MS; 2010.

2. Anhas DM, Castro-Silva CR. Sentidos atribuídos por adolescentes e jovens à saúde: desafios da Saúde da Família em uma comunidade vulnerável de Cubatão, São Paulo, Brasil. Saúde Soc 2017; 26(2):484-495.

3. Recife. Secretaria de Saúde (SS). Secretaria Executiva de Coordenação Geral, Gerência Geral de Planejamento. Plano Municipal de Saúde 2018 - 2021. Recife: SS; 2018.

4. Barros RP, Holanda PRCM, Silva ADS, Apostolico MR. Adolescente e adolescência: compreensão das necessidades em saúde para atenção integral. Atas do $8^{\circ}$ Congresso Iberoamericano de Investigação Qualitativa. CIAIQ. 2019. Investigação Qualitativa em Saúde, Volume 2. p. 1627-1636

5. Tong A, Sainsbury P, Craig J. Consolidated criteria for reporting qualitative research (COREQ):a 32-item checklist for interviews and focus groups. Int J Qual Health Care 2007; 19(6):349-357.

6. Egry EY. Saúde coletiva: construindo um novo método em Enfermagem. São Paulo: Ícone; 1996.

7. Egry EY. Metodologias para captação da realidade objetiva. In: Egry EY, organizadora. As necessidades em saúde na perspectiva da atenção básica: guia para pesquisadores. São Paulo: Dedone; 2008. p. 79-90.

8. Schraiber LB, Mendes-Gonçalves RB. Necessidades de saúde e atenção primária. In: Schraiber LB, Nemes MIB, Mendes-Gonçalves RB, organizadores. Saúde de adulto: programas e ações na unidade básica. São Paulo, Hucitec; 2000. p. 29-47. 
9. Paim JS. Desafios para a saúde coletiva no Século XXI. Salvador: Ed. UFBA; 2006.

10. Bardin L. Análise de conteúdo. São Paulo: Edições 70; 2001.

11. Tomasi E, Fernandes PAA, Fischer T, Siqueira FCV, Silveira DS, Thume E, Duro SMS, Saes MO, Nunes BP, Fassa ACG, Facchini LA. Qualidade da atenção pré-natal na rede básica de saúde do Brasil: indicadores e desigualdades sociais. Cad Saude Publica 2017; 33(3):e00195815.

12. Santos M, Alencar A, Lima S, Silva G, Carvalho C, Farre A, Sousa L. Educational pre-carnival on sexually transmitted infections with school adolescents. Journal of Nursing UFPE on line 2017; 11(12):5116-5121.

13. Hooper SS. Geração e juventude: o debate sobre a geração AI-5. Projeto História 2017; 59:337-362.

14. Pessalacio JDR, Menezes ES, Massuia E. A vulnerabilidade do Adolescente numa perspectiva das Políticas de Saúde Pública. BioEthikos 2010; 4(4):423-430.

15. Gessner R, Fonseca RMGS, Oliveira RNG. Violência contra adolescentes: uma análise à luz das categorias gênero e geração. Rev Esc Enferm USP 2014; 48(n. esp.):102-108.

16. Rytter D, Rask, CU, Vestergaard CH, Nybo Andersen AM, Bech BH. Non-specific Health complaints and self-rated health in pre-adolescents; impact on primary health care use. Sci Rep 2020; 10:3292.

17. Reis DC, Alves RH, Jordão NAF, Viegas AM, Carvalho SM. Vulnerabilidades e acesso em saúde na adolescência na perspectiva dos pais. Rev. Pesqui. Cuid. Fundam. 2014; 6(2):594-606.

18. Luz RT; Coelho EAC, Teixeira MA, Barros AR, Carvalho MFAA, Almeida MS. Estilo de vida e a interface com demandas de saúde de adolescentes. REME Rev min enferm. 2018; 22:e-1097.

19. Martins MMF, Aquino R, Pamponet ML, Pinto Junior EP, Amorim LDAF. Acesso aos serviços de atenção primária à saúde por adolescentes e jovens em um município do Estado da Bahia, Brasil. Cad Saude Publica 2019; 35(1):e00044718.

20. Oliveira RNG, Gessner R, Brancaglioni BCA, Fonseca RMGS, Egry EY. A prevenção da violência por parceiro(a) íntimo(a) na adolescência: uma revisão integrativa. Rev Esc Enferm USP 2016; 50(1):134-143.

21. Nunes GP, Sena FG, Costa CC, Kerber NPC, Zanchi M, Gonçalves CV. Gestante Adolescente e seu sentimento acerca do apoio familiar. Rev. Enferm. UFSM 2018; 8(4):1-13.

22. Lima CA, Moreira KS, Costa GS, Maia RS, Pinto MQC, Vieira MA, Costa SM. Avaliação do processo de trabalho entre equipes de saúde da família em um município de Minas Gerais, Brasil. Trabalho, Educação e Saúde 2019; 17(1):e0018710.
23. Zhou W, Ouyang F, Nergui OE, Bangura JB, Acheampong K, Massey IY, Xiao S. Child and Adolescent Mental Health Policy in Low- and Middle-Income Countries: Challenges and Lessons for Policy Development and Implementation. Front. Psychiatry 2020; 11:150.

24. Burgess T, Braunack-Mayer A, Tooher R, Collins J, O’Keefe M, Skinner R, Watson M, Ashmeade H, Proeve C, Marshall H. Optimizing intersectoral collaboration between health and education: the Health Bridges study. J Public Health 2015; 38(4):e430-e437.

25. Brasil EGM, Silva RM, Silva MRF, Rodrigues DP, Queiroz MVO. Promoção da saúde de adolescentes e Programa Saúde na Escola: complexidade na articulação saúde e educação. Rev. Esc. Enferm. USP 2017; 51:e03276.

26. Araújo WA, Sousa JCM, Medeiros RLSFM, Silva EN, Araujo IS, Carvalho FO, Assis EV, Feitosa ANA. Processo de trabalho e planejamento das ações de saúde. Rev Enferm UFPE 2018; 12(10):2564-2572.

27. Garuzi M, Achitti MCO, Sato CA, Rocha SA, Spagnuolo RS. Acolhimento na Estratégia Saúde da Família: revisão integrativa. Rev Panam Salud Publica 2014; 35(2):144-149.

28. Biffi D, Melo MFR, Ribeiro VR. Acolhimento de enfermagem á saúde do adolescente em uma estratégia de saúde da família. R. Perspect. Cie Saúde 2018; 3(1):8397.

29. Rodrigues-Morais R, Couto MCV, Faria DLS, Modena $\mathrm{CM}$. O atendimento à crise e urgência no Centro de Atenção Psicossocial para crianças e adolescentes de Betim. Pesquisas e Práticas Psicossociais 2019; 14(1):115.

30. Queiroz MKS, Rodrigues ILA, Nogueira LMV, Silva IFS. Fluxos assistenciais e a integralidade da assistência à saúde de ribeirinhos. Rev Enferm UERJ 2018; 26:e26706.

Artigo apresentado em 13/04/2020

Aprovado em 28/05/2020

Versão final apresentada em 30/05/2020

Editores chefes: Maria Cecília de Souza Minayo, Romeu Gomes, Antônio Augusto Moura da Silva 